\title{
The Role of POPDC1 in the Progression of the Malignant Phenotype
}

\author{
Steve Tucker ${ }^{1}$ and Alina Zorn ${ }^{1}$ \\ ${ }^{1}$ University of Aberdeen
}

September 22, 2020

\begin{abstract}
The Popeye Domain Containing Protein 1 (POPDC1), a tight junction-associated transmembrane protein with a unique binding site for cAMP, has been shown to act as a tumour suppressor in cancer cells. Through interaction with many downstream effectors and signalling pathways, POPDC1 promotes cell adhesion and inhibits uncontrolled cell proliferation, epithelial-tomesenchymal transition, and metastasis. However, POPDC1 expression is downregulated in many types of cancer, thereby reducing its tumour-suppressive actions. This review discusses the role of POPDC1 in the progression of the malignant phenotype and highlights the broad range of benefits POPDC1 stabilisation may achieve therapeutically. Cancer stem cells (CSC) are a key hallmark of malignancies and commonly promote treatment resistance. This article provides a comprehensive overview of CSC signalling mechanisms, many of which have been shown to be regulated by POPDC1 in other cell types, thus suggesting an additional therapeutic benefit for POPDC1-stabilising anticancer drugs.
\end{abstract}

\section{Hosted file}

Zorn and Tucker_The Role of POPDC1 in the Progression of the Malignant Phenotype_MAIN FILE.pdf available at https://authorea.com/users/361045/articles/482555-the-role-of-popdc1-in-theprogression-of-the-malignant-phenotype 


\begin{tabular}{|c|c|c|c|}
\hline & POPDC2 & POPDC3 & Reference \\
\hline $\begin{array}{c}\text { Cardiovascular } \\
\text { Disease }\end{array}$ & $\begin{array}{l}\text { Under-expressed causing } \\
\text { decreased conduction and } \\
\text { AV block in cardiac muscle } \\
\text { only. Associated with } \\
\text { W188X mutation. }\end{array}$ & - & $\begin{array}{l}\text { Brand, } 2019 \\
\text { Amunjela et al., } \\
2019\end{array}$ \\
\hline $\begin{array}{l}\text { Limb Girdle } \\
\text { Muscular } \\
\text { Dystrophy } \\
\text { (LGMD) }\end{array}$ & - & $\begin{array}{l}\text { Mutant (L155H, L217F, } \\
\text { R261Q) POPDC3 } \\
\text { expression leads to } \\
\text { skeletal muscular } \\
\text { dystrophy. }\end{array}$ & $\begin{array}{l}\text { Vissing et al., } \\
2019\end{array}$ \\
\hline $\begin{array}{c}\text { Ductal Breast } \\
\text { Carcinoma } \\
\text { (especially } \\
\text { HER2+ subtype) }\end{array}$ & $\begin{array}{l}\text { Over-expressed at all } \\
\text { clinical stages. Possibly } \\
\text { implicated in cancer } \\
\text { initiation and sustenance. }\end{array}$ & $\begin{array}{l}\text { Over-expressed at early } \\
\text { clinical stages. }\end{array}$ & $\begin{array}{l}\text { Amunjela and } \\
\text { Tucker, 2017a }\end{array}$ \\
\hline $\begin{array}{l}\text { Head and Neck } \\
\text { Squamous Cell } \\
\text { Carcinoma } \\
\text { (HNSCC) }\end{array}$ & - & $\begin{array}{l}\text { Over-expression } \\
\text { correlates with low } \\
\text { patient survival. Potential } \\
\text { biomarker for radio } \\
\text { therapy resistance. }\end{array}$ & He et al., 2019 \\
\hline Gastric Cancer & - & $\begin{array}{l}\text { Under-expression due to } \\
\text { promoter } \\
\text { hypermethylation. Lower } \\
\text { POPDC3 levels correlate } \\
\text { with increased depth of } \\
\text { invasion and metastasis. }\end{array}$ & $\begin{array}{l}\text { Lue et al., } 2012 \\
\text { Kim et al., } 2010\end{array}$ \\
\hline $\begin{array}{c}\text { Oesophageal } \\
\text { and Lung Cancer }\end{array}$ & - & $\begin{array}{l}\text { Overexpression of } \\
\text { POPDC3 correlates with } \\
\text { greater radiotherapy } \\
\text { resistance. }\end{array}$ & He et al., 2019 \\
\hline
\end{tabular}

Table 1 - The Role of POPDC2 and POPDC3 in Disease

Expression of POPDC2 and POPDC3 varies between tissue type and across various cancer types. Dysregulation of POPDC2 is mainly observed in cardiovascular disease and breast cancer. POPDC3 mutations are implicated in limb girdle muscular dystrophy and has been shown to have both tumour-suppressive and oncogenic roles in different malignancies. 


\begin{tabular}{|c|c|c|c|c|}
\hline Protein & $\begin{array}{l}\text { Interacting } \\
\text { Sequence of } \\
\text { POPDC1 }\end{array}$ & $\begin{array}{l}\text { Tissue location of } \\
\text { POPDC1 interaction }\end{array}$ & Suggested Role & References \\
\hline TREK-1 & $\begin{array}{c}\text { Unknown sequence } \\
\text { on CTD }\end{array}$ & Cardiac myocytes & $\begin{array}{l}\text { Interaction with POPDC1 } \\
\text { enhances current flow in } \\
\text { cardiac myocytes }\end{array}$ & $\begin{array}{l}\text { Han et ol., } \\
2019 \\
\text { Brand, } 2019\end{array}$ \\
\hline CAV-3 & aa242-266 & $\begin{array}{c}\text { Skeletal muscle } \\
\text { sarcolemma, cardiac } \\
\text { myocyte transverse } \\
\text { tubules }\end{array}$ & $\begin{array}{l}\text { POPDC1 ensures } \\
\text { structural integrity and } \\
\text { function of Cav-3 }\end{array}$ & $\begin{array}{l}\text { Han et ol., } \\
2019 \\
\text { Brand, } 2019\end{array}$ \\
\hline VAMP3 & $\begin{array}{c}\text { CTD sequence after } \\
\text { aa118 }\end{array}$ & $\begin{array}{l}\text { MDCK cells, adult } \\
\text { cardiac and skeletal } \\
\text { muscle }\end{array}$ & $\begin{array}{l}\text { POPDC1 interaction } \\
\text { ensures adequate } \\
\text { recycling of } \beta 1 \text {-integrins. } \\
\text { Loss of this interaction } \\
\text { increases migration. }\end{array}$ & $\begin{array}{l}\text { Hager et al., } \\
2010\end{array}$ \\
\hline GEFT & aa250-300 & $\begin{array}{l}\text { Human Corneal } \\
\text { Epithelia, Murine } \\
\text { NIH T3T Cells }\end{array}$ & $\begin{array}{l}\text { Retention of GEFT in } \\
\text { membrane, preventing } \\
\text { Rac1/Cdc42/RhoA } \\
\text { activation promoting TJ } \\
\text { formation }\end{array}$ & $\begin{array}{c}\text { Russ et al., } \\
\text { 2010, Smith et } \\
\text { al., } 2008\end{array}$ \\
\hline GEFH & $\begin{array}{c}\text { Unknown sequence } \\
\text { on CTD }\end{array}$ & $\begin{array}{l}\text { Human Corneal } \\
\text { Epithelia }\end{array}$ & $\begin{array}{l}\text { POPDC1 sequesters GEFH } \\
\text { to cell membrane to } \\
\text { prevent RhoA signalling }\end{array}$ & $\begin{array}{l}\text { Russ et al., } \\
\text { 2011, Parang } \\
\text { et al., } 2018\end{array}$ \\
\hline zo-1 & $\begin{array}{c}\text { Unknown sequence } \\
\text { on CTD }\end{array}$ & $\begin{array}{c}\text { Trabecular } \\
\text { meshwork cells, } \mathrm{HCE} \text {, } \\
\text { uveal melanoma }\end{array}$ & $\begin{array}{l}\text { POPDC1/ZO-1 interaction } \\
\text { prevents ZONAB-induced } \\
\text { entry to cell cycle and } \\
\text { translation of } \\
\text { proliferative genes }\end{array}$ & $\begin{array}{c}\text { Russ et al., } \\
\text { 2010, Russ et } \\
\text { al., } 2011 \\
\text { Amunjela et } \\
\text { al., 2019, } \\
\text { Jayagopal et }\end{array}$ \\
\hline Occludin & $\begin{array}{l}\text { Unknown sequence } \\
\text { on CTD }\end{array}$ & $\begin{array}{l}\mathrm{HCE} \text {, uveal } \\
\text { melanoma }\end{array}$ & $\begin{array}{c}\text { Maintenance of tight } \\
\text { junction formation }\end{array}$ & al., 2011 \\
\hline Bnip3 & $\begin{array}{c}\text { Unknown sequence } \\
\text { on CTD }\end{array}$ & Cardiac Myocytes & $\begin{array}{c}\text { POPDC1 suppresses } \\
\text { Bnip3-induced apoptosis }\end{array}$ & $\begin{array}{c}\text { Kliminski et al., } \\
2016\end{array}$ \\
\hline $\begin{array}{l}\text { LRP6 } \\
\text { (Wnt/B- } \\
\text { catenin- } \\
\text { pathway) }\end{array}$ & $\begin{array}{c}\text { Unknown sequence } \\
\text { on CTD }\end{array}$ & $\begin{array}{c}\text { HEK293 cells, } \\
\text { human colonoids, } \\
\text { murine adenoma } \\
\text { tumoroids }\end{array}$ & $\begin{array}{l}\text { Prevention of } \beta \text {-catenin } \\
\text { activation by inhibition of } \\
\text { LRP6 }\end{array}$ & $\begin{array}{c}\text { Thompson et } \\
\text { al., } 2019\end{array}$ \\
\hline $\begin{array}{l}\text { PR61a (c- } \\
\text { Myc } \\
\text { pathway) }\end{array}$ & aa330-345 & $\begin{array}{l}\text { Murine colitis- } \\
\text { associated cancer } \\
\text { cells }\end{array}$ & $\begin{array}{c}\text { Promotes c-Myc } \\
\text { ubiquitination / } \\
\text { degradation }\end{array}$ & $\begin{array}{l}\text { Parang et al-, } \\
2017\end{array}$ \\
\hline
\end{tabular}

Table 2-POPOC1 Downstream Targets

The POPDC1 pratein interacts with many dewnstream including TREK1, CAV-3, VAMP3, GEFT, GEFH, 20-1, accludin, Bnip 3, LRPE and PR61a. This interaction has mainly been shown in cardiac and skeletal muscle cells, however, an increasing body of evidence is emerging that demonstrates POPDC1 interaction with these targets in cancer cells.

Abbreviations: CTD (C-terminal domain), HCE (human corneal epithelial cells), HEK293 (human embryonic kidney cells) 


\begin{tabular}{lcl}
\hline \multicolumn{1}{c}{$\begin{array}{c}\text { Mechanisms of POPDC1 } \\
\text { Downregulation }\end{array}$} & Cancer type & \multicolumn{1}{c}{ Reference } \\
\hline Promoter Hypermethylation & CRC, PC, BC, NSCLC, Glioma, \\
HNSCC, GC & $\begin{array}{l}\text { Williams et al., 2010; Parang et } \\
\text { al., 2017, Amunjela and } \\
\text { Tucker, 2016; Kim et al., 2010. }\end{array}$ \\
\hline $\begin{array}{l}\text { Under-expression of miRNA- } \\
\mathbf{1 2 2}\end{array}$ & HCC & Wang et al., 2014 \\
\hline Over-expression of Netrin-1 & HCC & Han et al., 2015 \\
\hline EGFR activation & BC & Amunjela and Tucker, 2017a \\
\hline
\end{tabular}

Table 3-Mechanisms of POPDC1 Downregulation Associated with Various Cancer Types

The four main mechanisms of PODPC1 downregulation include promoter hypermethylation, under-expression of miRNA122 reducing POPDC1 gene transcription, over-expression of netrin-1 leading to inhibited POPDC1 expression and EGFR activation, which phosphorylates and inactivates POPDC1. These mechanisms have been observed in many different cancer types including HCC, CRC, BC, PC, NSCLC, HNSCC and glioma. The most commonly identified mechanism of POPDC1 downregulation is promoter hypermethylation.

Abbreviations: CRC (colorectal cancer), PC (prostate cancer), BC (breast cancer), NSCLC (non-small cell lung cancer), HNSCC (head and neck squamous cell carcinoma), GC (gastric cancer), HCC (hepatocellular carcinoma). 


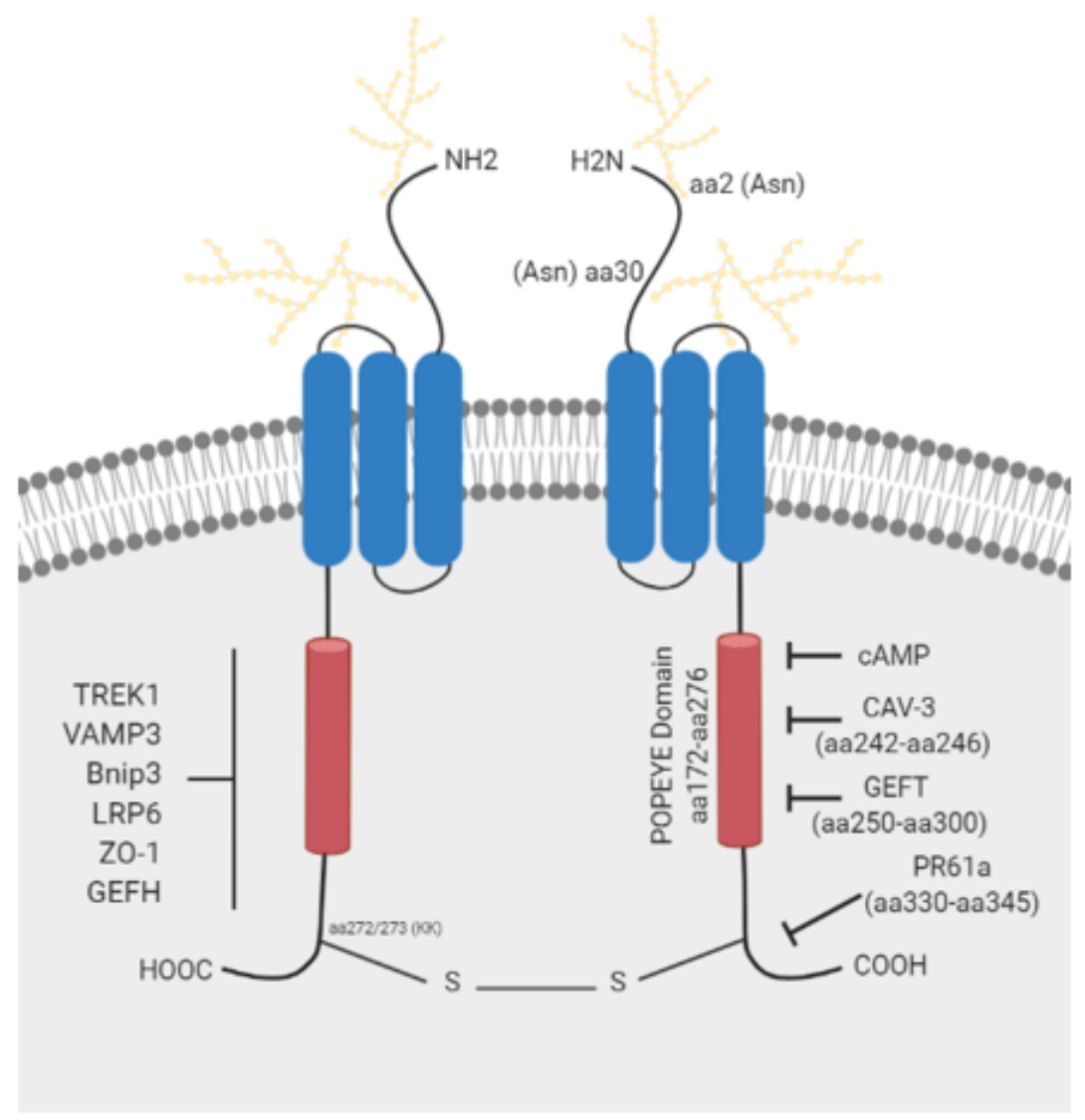

Figure 1 - The Structure of Popeye Domain Containing Protein 1 (POPDC1)

POPDC1 is a transmembrane protein highly expressed in cardiac myocytes, skeletal muscle and some cancer cells. The short extracellular $\mathrm{N}$-terminal domain contains two glycosylation sites (asparagine residues at positions aa2 and aa30). This is followed by three membrane-spanning domains and a long intracellular $\mathrm{C}$ terminal domain (CTD). The CTD contains the highly conserved POPEYE domain (aa172-aa276) with a unique CAMP binding site. Other binding sites exist for TREK1, VAMP3, Bnip3, LRP6, ZO-1, GEFH, CAV-3 (aa242-246), GEFT (aa250-300) and PR61a (aa330-aa345). A disulfide bridge forms between intracellular cysteine residues with lysine residues (aa272/273) critically required for this dimerisation, which promotes membrane stabilisation.

Image created with BioRender.com 


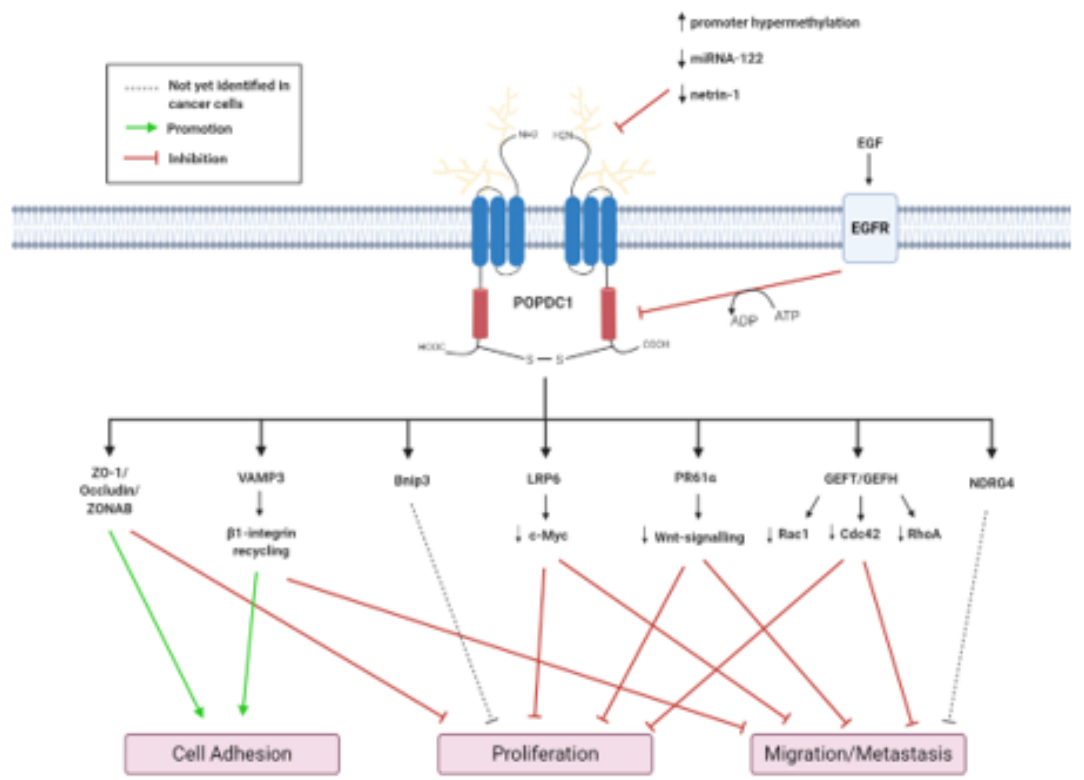

Figure 2 - Regulatory Roles of POPOC1 in Cancer

POPDC1 acts as a tumour suppressor by influencing three main processes involved in cancer progression. its interaction with tight junction-associated proteins (e.g. 20-1, accludin, ZONAB) and VAMP3 ensures maintenance of cell adhesion. Furthermore, POPDC1 inhibits uncontrolled cell proliferation through its interactions with guanine nucleotide exchange factors (GEFT/GEFH) and the C-Myc and Wht-signalling pathways. POPDC1 also plays an important rale in suppressing cancer cell migration and metastasis.

Lass of these regulatory roles exerted by POPDC1 can occur as aseswlt of intracellular phosphorylation (through EGFR) or reduced gene expressian due to promater hypermethylation, reduced miRNA-122 expression or increases netrin-1 activity.

Image created with BioRender.com 